\title{
Journal of Surgery
}

\section{Isolated Celiac Trunk Dissection after Cardiac Surgery}

\author{
Francesco Terrieri' ${ }^{1}$, Marzia Cottini ${ }^{2 *}$, Marco Picichè ${ }^{2}$, Stefano Rausei ${ }^{3}$ and Cesare Beghi ${ }^{1}$ \\ ${ }^{1}$ Cardiac Surgery Unit and Heart Transplantation Centre, Department of Heart and Vessels, "S. Camillo-Forlanini" Hospital, Italy \\ ${ }^{2}$ Cardiac Surgery Unit, Department of Cardiac Surgery, University Hospital Circolo Fondazione Macchi, Italy \\ ${ }^{3}$ General Surgery Unit, Department of General Surgery, University Hospital Circolo Fondazione Macchi, Italy
}

\begin{abstract}
The most catastrophic postoperative gastrointestinal complication in cardiac surgery is mesenteric ischemia, which is frequently fatal. This may result from atheroembolization, heparin-induced thrombocytopenia or hypoperfusion. We reported the case of 72 year old man undergone to coronary artery bypass and aortic valve replacement, presented isolated celiac trunk dissection after surgery. CT scan described a dissection flap of the first part of celiac trunk possible due to ulcerative plaque without note of anterograde or retrograde development. Considering patient's hemodynamic, respiratory and general trend, we decided to choose conservative management. The sequent postoperative period was uneventfully, the patient was hemodynamically stable and he was discharged at the $20^{\text {th }}$ postoperative day. The atheroembolization was a life-threatening problem of all vascular districts: a correct CPB pressure and postoperative BP monitoring could help to reduce the incidence of its complications.
\end{abstract}

Keywords: Complication cardiac surgery; Celiac trunk dissection; Post cardiac surgery

\section{Introduction}

Spontaneous dissection of the superior mesenteric and inferior mesenteric arteries and of the celiac artery is uncommon occurrence [1]. Celiac artery dissection (CTD) is rare, and can present with intimal flap, mural thrombus or infiltration of fat around the artery. The causes of CTD are several and different: hypertension, arteriosclerosis, degeneration of the arterial wall, trauma, pregnancy, and arteriopathy, iatrogenic and unknown ones. The evolution of CTD isn't well known: it can be associated with complications like aneurysm formation or occlusion, splenic infarction, intraperitoneal hemorrhage and the intestinal ischemia. There are several management modalities applied by the physicians on a case by-case basis like endovascular repair or interventional radiological approach [2] or conservative treatment options by anticoagulants and anti-platelets besides antihypertensive drug are also applied. The choice of CTD isn't univocal and weedefined: according to the reported scientific paper documented CTD from 1959, the proper treatment is related to celiac trunk lesion, celiac trunk complication and patient's clinical trend. The medical treatment consists of oral anticoagulation therapy and monitoring of the patient. The medical therapy failure or the CTS complicated with widespread bowel ischemia and /or large splenic infarction needs surgery because the life-threatening development. On the contrary, a localized and mild CTD due to malperfusion or with high risk of rupture could be treated by endovascular choice.

\section{Case Report}

A 72 year old male with a history of paroxysmal atrial fibrillation and chronic coronary artery disease treated with percutaneous coronary intervention two years earlier, was admitted to our hospital suffering from chest pain (angina) and exercise intolerance. Coronary angiography documented a severe lesion of the main stem $(>70 \%)$ and mild lesion (50\%) of the proximal right coronary artery. A Trans Thoracic Echocardiography (TTE) revealed mildly dilated left ventricle (LVEF 40\% and LV-End diastolic Volume $120 \mathrm{ml} / \mathrm{m}^{2}$ ) and moderate aortic regurgitation (Regurgitation Volume $35 \mathrm{ml} /$ beat).

He underwent double coronary artery bypass surgery and Aortic Valve Replacement (AVR, with bioprosthetic valve). Surgery was performed with standard approach (median sternotomy) in normothermic $\left(34^{\circ} \mathrm{C}\right)$ extracorporeal circulation. The Cardiopulmonary Bypass (CPB) time was 170 minutes and mean blood pressure was maintained at about $70 \mathrm{~mm} \mathrm{Hg}$. The perioperative period was free of early complications. Hence, we administered Indobufen (200 mg die) and Warfarin to achieve a International Number Ratio (INR) range between 2 to 3 . At the second postoperative day, the patient suffered two episodes of high-rate atrial fibrillation, immediately treated with Amiodarone and Metoprolol After 48 hours, the patient presented abdominal pain with negative physical examination (the pain was mild and widespread, epigastric tenderness, no organomegaly, no signs of ascites, no abdomen aortic pulsation, normal bowel sounds, Blumberg', Murphy' and Giordano signs were negative) but mild leukocytosis (WBC $11980 / \mathrm{mm}^{3}$ ). So we started diagnostic workup: an abdomen X-ray showed small airfluid levels in the jejunal; abdominal ultrasonography was negative; a 64-slice computed tomography (CT) scan showed dissection of the celiac branch of the abdominal aorta (Figure 1A and B) associated with mesenteric distension. Hereby, an endoscopic exam documented ulceration with necrotic area at the second portion of duodenum without evident bleeding sites. Considering the patient details (Table 1) and the high surgical risk due to recent cardiac surgery and possible mesenteric ischemia, we collegially decided for conservative therapy. The patient was monitored in the intensive care unit, nasogastric tube was positioned and antibiotic prophylaxis was started. Indobufen and Warfarin were stopped and we introduced low molecular weight heparin (Enoxaparin 4000 UI subcutaneous twice daily) with proton pump inhibitors (PPI, Pantoprazole $40 \mathrm{mg}$ die intravenous). Two days later, a 64-slide CT scan was performed and showed no aggravation or modification of the abdominal view (Figure 2). At the $12^{\text {th }}$

*Corresponding author: Marzia Cottini, Department of Heart and Vessels, Cardiac Surgery Unit, "S. Camillo- Forlanini" Hospital, 00149 Rome, Italy, Tel: +39-3473245331; Fax: +39-06-58704511; E-mail: marzia.cottini@hotmail.it

Received February 29, 2016; Accepted May 11, 2016; Published May 18, 2016

Citation: Terrieri F, Cottini M, Picichè M, Rausei S, Beghi Beghi. Isolated Celiac Trunk Dissection after Cardiac Surgery. Journal of Surgery [Jurnalul de chirurgie]. 2016; 12(2): 71-73 DOI:10.7438/1584-9341-12-2-6

Copyright: $\odot 2016$ Terrieri F, et al. This is an open-access article distributed unde the terms of the Creative Commons Attribution License, which permits unrestricted use, distribution, and reproduction in any medium, provided the original author and source are credited. 

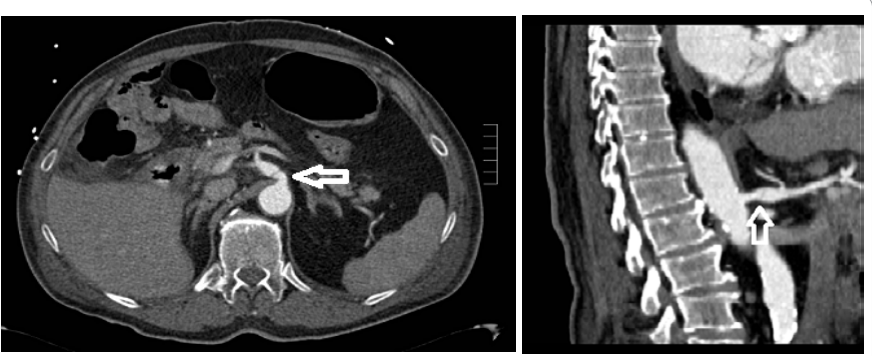

Figure 1A: Axial contrast-enhanced CT image shows celiac trunk proximal partial dissection. 1B: Sagittal contrast-enhanced CT abdomen/pelvis documents the dissection of celiac trunk.

Table I: Patient's trend details after the $2^{\text {nd }}$ postoperative day of cardiac surgery.

\begin{tabular}{|c|c|}
\hline \multicolumn{2}{|c|}{ Celiac Trunk Dissection After CS: Patient's Trend Details } \\
\hline Mild Symptoms & Widespread and Shallow Abdominal Pain \\
\hline Mild diagnostic signs & $\begin{array}{l}\text { 1. Abdomen X-ray showed small air-fluid levels in the } \\
\text { jejunal; } \\
\text { 2. Abdominal ultrasonography was negative } \\
\text { 3. CT scan showed local and limited dissection of the } \\
\text { celiac branch of the abdominal aorta } \\
\text { 4. Endoscopic exam documented an ulceration with } \\
\text { necrotic area at the second portion of duodenum without } \\
\text { evident bleeding sites }\end{array}$ \\
\hline $\begin{array}{l}\text { Stable hemodynamic } \\
\text { trend }\end{array}$ & Optimal blood pressure, PAM $(80 / 85 \mathrm{mmHg})$ \\
\hline $\begin{array}{l}\text { Optimal respiratory } \\
\text { trend }\end{array}$ & $\begin{array}{l}\text { Normal value of } \mathrm{PaO}_{2}, \mathrm{PCO}_{2} \text {, mean } \mathrm{pH} 7.38 \text {, mean } \mathrm{HCO}_{3} \\
24 \mathrm{mEq} / \mathrm{L}, \mathrm{BE}-0.6, \mathrm{SaO}_{2} 98 \% \text {, mean CVP } 8 \text {. }\end{array}$ \\
\hline $\begin{array}{l}\text { Stable celiac trunk } \\
\text { lesion }\end{array}$ & $\begin{array}{l}\text { Documented by: } \\
\text { 1. CT scans performing in the } 2^{\text {nd }}, 4^{\text {th }} \text { and } 12^{\text {th }} \\
\text { postoperative day. } \\
\text { 2. No signs and symptoms of sub-ileus and intestinal } \\
\text { obstruction }\end{array}$ \\
\hline
\end{tabular}

3. No signs and symptoms of gastrointestinal bleeding.

Note: MAP: Mean Arterial Pressure; CVP: Central Venous Pressure; $\mathrm{PaO}_{2}$ : Partial Arterial Pressure of Oxygen; $\mathrm{PaCO}_{2}$ : Partial Arterial Pressure of Carbon Dioxide CT: Computed Tomography; $\mathrm{SaO}_{2}$ : Arterial Oxygen Saturation

postoperative day, the endoscopy showed worsening and increasing of the ulcerations at the second segment of the duodenum with an evident bleeding site, ulcerations were treated with endoclips and local therapeutic injection of Epinephrine (1:10.000, $8 \mathrm{ml})$. The subsequent course was uneventful and with stable haemoglobin at laboratory test. At the $20^{\text {th }}$ postoperative days, the US-ECHO did not detect the intimal flap of celiac branch (Figure $3 \mathrm{~A}$ and $\mathrm{B}$ ). The endoscopy showed a regression of ulcerative lesions of the duodenum (re-epithelialisation). The patient was discharged and the 1 year and 3 year follow up was free of long-term complication. He didn't refer any abdominal symptoms, the control CT scan and echography didn't showed alteration of the abdomen vascularisation (Figure 4).

\section{Discussion}

Gastrointestinal complications after cardiac surgery are rare but carry significant morbidity and mortality even when recognized early and treated appropriately. Reviewing the scientific literature these complications are rare $(0.53 \%-2.1 \%)$ but often fatal $(13.9 \%$ 63\%) [3]. According to Mangi et al. [4], the documented or suspected postoperative mesenteric ischemia is a life-threatening fatal complication (death in the $68 \%$ of the cases). The outcome in mesenteric ischemia is closely dependent on the elapsed time to diagnosis and treatment. The diagnosis is typically difficult and delayed due to nonspecific results of biological and radiological tests. The etiology of mesenteric ischemia may also be conceptually classified into two distinct subsets: low flow and low perfusion states and embolic related events. Mangi et al. [4] observed that CPB had been implicated in the development of mesenteric ischemia modifying the regional intestinal blood flow, by pulsating to continuous flow. Tao et al. [5] demonstrated that normothermic CPB may cause a diversion of the blood flow in the splanchnic district away from the ileum and colon. These changes accompanied dysfunction of mesenteric endothelial cells causing hyper-reactivity of alpha agonists [6]. Survival from the insult of mesenteric ischemia depends partially upon the early diagnosis. The most sensitive indicators of mesenteric ischemia after cardiac surgery are abdominal pain (continuous, not local), increasing of lactate and base excess (BE) and spreading of metabolic acidosis. As the majority of cases of mesenteric ischemia occur during the initial hours to days following operation, fast-track extubation pathways and or minimal sedation theoretically may enable earlier recognition of a catastrophic gastrointestinal complication. When intestinal ischemia is clinically suspected, diagnostic imaging studies should be performed even if peritoneal signs are absent.

Acute mesenteric ischemia is considered a vascular emergency; if treated during its initial stage, its mortality rate is less than $30 \%$ but if treatment is begun more than 6 to 8 hours after symptom onset, the mortality rate increases exponentially. Various treatment options are currently available, including conservative management, anticoagulation, endovascular stenting, and surgical repair. A review of current literature show that endovascular treatment techniques should be preferred, in order to place less of an additional burden on patients, most of whom have multiple morbidities $[7,8]$; immediate laparotomy must be performed for acute abdomen and revascularization of the intestine is the primary aim of treatment. Irreversibly damaged portions of the intestine must be resected.

Carefully analyzing our reported case report (Table 1), we decided collegially (cardiac surgeon, cardiologist, cardiovascular anaesthesist and general surgeon) to choice the conservative management. Our conservative management consisted of: strict blood pressure monitoring, every day blood gas analysis and diuresis balance, daily control of bowel function and abdomen physical exam, performing control CT scan and abdomen echography periodically with a maximum interval time of 48 hours. Following this management, we have controlled closely the patient, every possible variables and changes, and the patient was successfully discharged after 20th day.

\section{Conclusion}

Acute mesenteric ischemia is a life-threatening surgical emergency in which the outcome is closely dependent on the elapsed time to diagnosis and treatment. Some studies have undertaken systematic analyses of preoperative, intraoperative, and postoperative variables to define the risk stratification to influence cardiac surgeons to

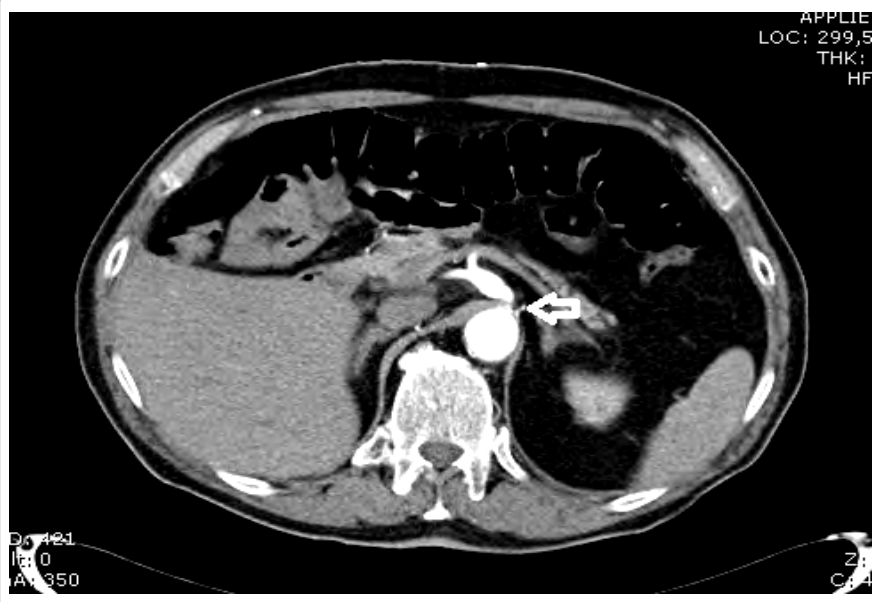

Figure 2: Axial contrast-enhanced CT abdomen/pelvis showing no worsening of celiac trunk dissection. 


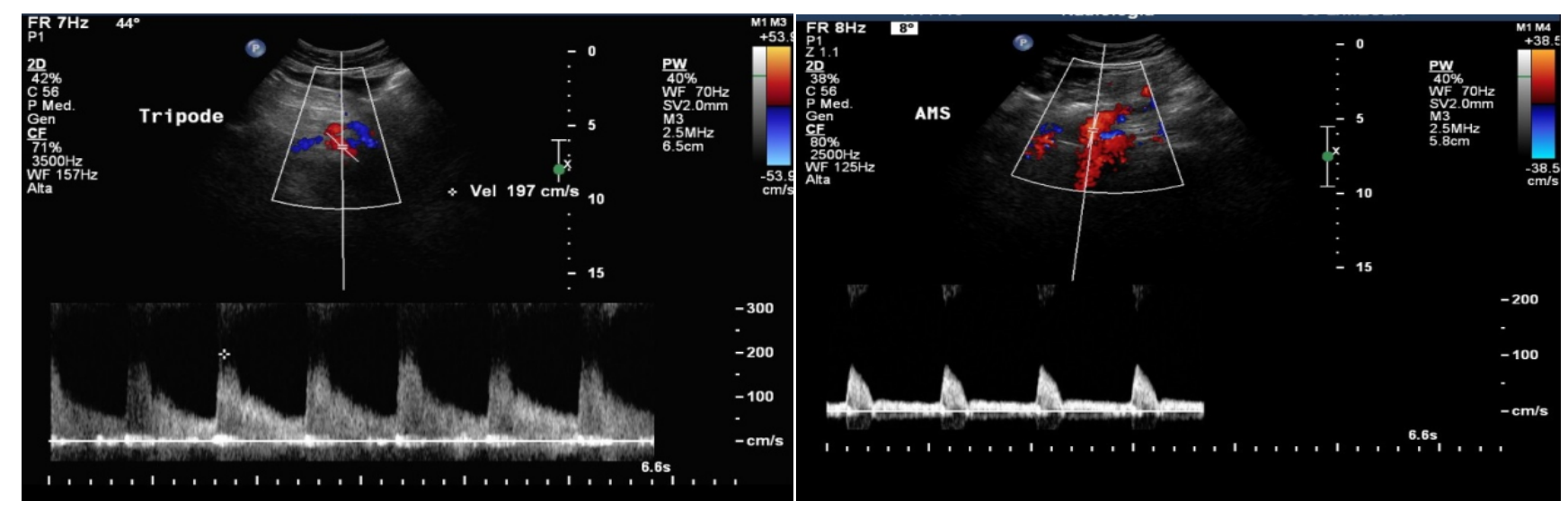

Figure 3A: Duplex Doppler sonography of celiac trunk. 3B: Duplex Doppler sonography of superior mesenteric artery.

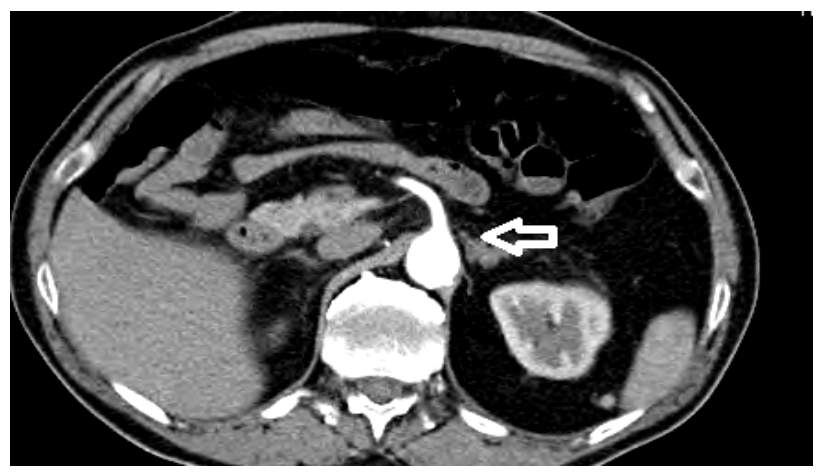

Figure 4: Axial contrast-enhanced CT abdomen/pelvis showing normal celiac trunk and abdomen perfusion.

alter their operative strategy or early postoperative management decisions. Identification of risk factors for death after gastrointestinal complications provides general surgeons with clinically relevant features indicating subsets of patients most likely to benefit from laparotomy. The outcome is closely dependent on the elapsed time to diagnosis and treatment. Conservative management consisted of strict blood pressure control, bowel rest, and close observation, without the use of anticoagulation or antiplatelet agents. Patients with a symptomatic dissection of the superior mesenteric artery should initially undergo conservative management without anticoagulation and antiplatelet therapy if bowel perfusion is not compromised and if the superior mesenteric artery is not likely to rupture. Surgery is indicated in patients with superior mesenteric artery thrombosis; questionable bowel viability, bowel necrosis or severe haemorrhage. Patients initially treated with conservative management should be monitored closely and should undergo a follow-up CT scan.

\section{Conflict of Interest}

The authors declared no potential conflicts of interest with respect to the research, authorship, and publication of this article.

\section{References}

1. D'Ambrosio N, Friedman B, Siegel D, Katz D, Newatia A, et al. (2007) Spontaneous isolated dissection of the celiac artery: CT findings in adults. Am J Roentgenol 188: 6-11.

2. Fenoglio L, Allione A, Scalabrino E, Alberto G, Benedetti V, et al. (2004) Spontaneous dissection of the celiac artery: a pitfall in the diagnosis of acute abdominal pain.Presentation of two cases. Dig Dis Sci 49: 1223-1227.

3. Tsiotos GG, Mullany CJ, Zietlow S, van Heerden JA, (1994) Abdominal complications following cardiac surgery. Am J Surg 167: 553-557.
4. Mangi AA, Christison-Lagay ER, Torchiana DF, Warshaw AL, Berger DL, et al. (2005) Gastrointestinal complications in patients undergoing heart operation. Ann Surg 241: 895-904.

5. Tao W, Zwischenberger JB, Nguyen TT, Vertrees RA, McDaniel LB, et al (1995) Gut mucosal ischemia during normothermic cardiopulmonary bypass results from blood flowredistribution and increased oxygen demand. J Thorac Cardiovasc Surg 110: 819-828.

6. Abboud B, Daher R, Boujaoude J (2008) Acute mesenteric ischemia after cardio-pulmonary bypass surgery. World J Gastroenterol 14: 5361-5370.

7. Oh S, Cho YP, Kim JH, Shin S, Kwon TW, et al. (2011) Symptomatic spontaneous celiac artery dissection treated by conservative management: serial imaging findings. Abdom Imaging 36: 79-82.

8. Sparks SR, Vasquez JC, Bergan JJ, Owens EL (2000) Failure of nonoperative management of isolated superior mesenteric artery dissection. Ann Vasc Sur 14: 105-109. 